\title{
Chern-Simons flows on Aloff-Wallach spaces and spin(7) instantons
}

\author{
Alexander S. Haupt, ${ }^{1, *}$ Tatiana A. Ivanova, ${ }^{2, \dagger}$ Olaf Lechtenfeld, ${ }^{1,3,}$ and Alexander D. Popov ${ }^{2, \S}$ \\ ${ }^{1}$ Institut für Theoretische Physik, Leibniz, Universität Hannover, Appelstraße 2, 30167 Hannover, Germany \\ ${ }^{2}$ Bogoliubov Laboratory of Theoretical Physics, JINR, 141980 Dubna, Moscow Region, Russia \\ ${ }^{3}$ Centre for Quantum Engineering and Space-Time Research, Leibniz Universität Hannover, \\ Welfengarten 1, 30167 Hannover, Germany \\ (Received 4 May 2011; published 27 May 2011)
}

Because of their explicit construction, Aloff-Wallach spaces are prominent in flux compactifications. They carry $G_{2}$ structures and admit the $G_{2}$-instanton equations, which are natural Bogomol'nyi-PrasadSommerfeld equations for Yang-Mills instantons on seven-manifolds and extremize a Chern-Simons-type functional. We consider the Chern-Simons flow between different $G_{2}$ instantons on Aloff-Wallach spaces, which is equivalent to spin(7) instantons on a cylinder over them. For a general SU(3)-equivariant gauge connection, the generalized instanton equations turn into gradient-flow equations on $\mathbb{C}^{3} \times \mathbb{R}^{2}$, with a particular cubic superpotential. For the simplest member of the Aloff-Wallach family (with 3-Sasakian structure) we present an explicit instanton solution of tanh-like shape.

DOI: 10.1103/PhysRevD.83.105028

\section{INTRODUCTION AND SUMMARY}

Yang-Mills theory in more than four dimensions naturally appears in the low-energy limit of superstring theory in the presence of D-branes. Also, heterotic strings yield heterotic supergravity, which contains supersymmetric Yang-Mills theory as a subsector [1]. Furthermore, natural Bogomol'nyi-Prasad-Sommerfield-type equations for gauge fields in dimension $d>4$, introduced in [2], also appear in superstring compactifications on spacetimes $M_{10-d} \times X^{d}$ as the condition for the survival of at least one supersymmetry in the low-energy effective field theory on $M_{10-d}$ [1]. These first-order Bogomol'nyi-PrasadSommerfield-type equations on $X^{d}$, which generalize four-dimensional Yang-Mills anti-self-duality, were considered e.g. in [3-9], and some of their solutions were found in [10-13].

In string/M-theory compactification, the most interesting dimensions seem to be $d=6,7$ or 8 , and the corresponding generalized anti-self-duality equations are, respectively, called the Hermitian-Yang-Mills equations [4], the $G_{2}$-instanton equations $[8,14]$, or the $\operatorname{spin}(7)$ instanton equations $[8,15]$. Most work on the abovementioned instanton equations has restricted its attention to Riemannian manifolds $X^{d}$ with holonomy group $\mathrm{SU}(3)$ for $d=6, G_{2}$ for $d=7$, or $\operatorname{spin}(7)$ for $d=8$, i.e. to integrable $G$ structures. However, if one is interested in string compactification with fluxes [16], one should consider nonintegrable $G$ structures (weak holonomy groups) instead. The torsion of the $G$ structure, which measures the failure to be integrable, is identified with the three-form field ("flux") of supergravity. Flux compactifications have

\footnotetext{
*Alexander.Haupt@itp.uni-hannover.de

†ita@theor.jinr.ru

Olaf.Lechtenfeld@itp.uni-hannover.de

§popov@theor.jinr.ru
}

PACS numbers: 11.15.Yc, 11.10.Kk, 11.27.+d, 12.10.-g

been investigated primarily for type II strings and $\mathrm{M}$ theory, but also in the heterotic theories, albeit to a lesser extent, despite their long history [17]. In particular, compactifications on Aloff-Wallach spaces $[18,19] X_{k, l}$ of dimension seven and cones $\mathcal{C}\left(X_{k, l}\right)$ over them were studied e.g. in [20-22]. The Yang-Mills equations on spin(7) manifolds of topology $\mathbb{R} \times X_{k, l}$ with cylindrical and conical metric are the subject of the present paper.

For any coprime pair of integers $(k, l)$, the Aloff-Wallach space $X_{k, l}$ is the coset $\mathrm{SU}(3) / \mathrm{U}(1)_{k, l}$ with $\mathrm{U}(1)_{k, l}=$ $\left\{\operatorname{diag}\left(e^{i(k+l) \varphi}, e^{-i k \varphi}, e^{-i l \varphi}\right)\right\} \quad[18,19]$. It carries a $G_{2}$ structure defined by a torsion three-form $\psi$ with the property that $d \psi$ is proportional to the Hodge-dual fourform $* \psi . G_{2}$ instantons extremize a Chern-Simons-type action functional on $X_{k, l}$. As an example, we describe the Abelian canonical connection on a line bundle over $X_{k, l}$. Next, we step up to eight dimensions via extending $X_{k, l}$ by a real line $\mathbb{R}$. Our $G_{2}$ instantons are the end points of a gradient flow along this line, which is described precisely by the $\operatorname{spin}(7)$-instanton equations [8] on $\mathbb{R} \times X_{k, l}$. The most general SU(3)-equivariant connection on a rank-3 complex vector bundle is parametrized by three complex and two real functions on $\mathbb{R}$. The $\operatorname{spin}(7)$-instanton equations reduce to gradient-flow equations for these functions, governed by a cubic superpotential $W$ with global $\mathrm{U}(1) \times$ $\mathrm{U}(1)$ symmetry. Interestingly, each function obeys a linear equation in the background of the others.

In order to be more explicit, we specialize to the case of $k=l=1$. We fix the metric moduli (up to a freedom of orientation) such that $X_{1,1}$ is 3 -Sasakian and $\mathcal{C}\left(X_{1,1}\right)$ is hyper-Kähler, i.e. its structure group reduces to $\mathrm{Sp}(2)$. We list all critical points and their Hessians and numerically find an instanton solution whose shape is close to the tanh function. The corresponding gauge configuration interpolates between different $G_{2}$ instantons on $X_{1,1}$. It is not obvious how to establish the existence of further instanton 
solutions. It would be interesting to extend and lift spin(7) instantons on a cylinder or a cone over AloffWallach spaces to classical solutions of heterotic M theory.

\section{ALOFF-WALLACH SPACES}

Group $S U(3)$.-Consider the group $\mathrm{SU}(3)$ with generators $\left\{I_{a}, I_{i}\right\}, a=1, \ldots, 6, i=7,8$, satisfying

$$
\left[I_{a}, I_{b}\right]=f_{a b}^{c} I_{c}+f_{a b}^{i} I_{i}, \quad\left[I_{i}, I_{a}\right]=f_{i a}^{b} I_{b}, \quad\left[I_{i}, I_{j}\right]=0,
$$

where the structure constants are

$f_{13}^{5}=f_{42}^{5}=f_{41}^{6}=f_{32}^{6}=-\frac{1}{2 \sqrt{3}}, \quad f_{12}^{7}=f_{34}^{7}=\frac{1}{2 \sqrt{3}}$,

$f_{56}^{7}=-\frac{1}{\sqrt{3}}, \quad f_{12}^{8}=-f_{34}^{8}=-\frac{1}{2}$,

plus those with cyclic permutations of indices in (2.2). The generators (2.1) of SU(3) can be chosen in the form

$I_{1}=\frac{1}{2 \sqrt{3}}\left(\begin{array}{ccc}0 & 0 & -1 \\ 0 & 0 & 0 \\ 1 & 0 & 0\end{array}\right), \quad I_{3}=\frac{1}{2 \sqrt{3}}\left(\begin{array}{ccc}0 & 1 & 0 \\ -1 & 0 & 0 \\ 0 & 0 & 0\end{array}\right)$,

$I_{5}=\frac{1}{2 \sqrt{3}}\left(\begin{array}{ccc}0 & 0 & 0 \\ 0 & 0 & 1 \\ 0 & -1 & 0\end{array}\right), \quad I_{2}=\frac{1}{2 \sqrt{3}}\left(\begin{array}{lll}0 & 0 & i \\ 0 & 0 & 0 \\ i & 0 & 0\end{array}\right)$,

$I_{4}=\frac{1}{2 \sqrt{3}}\left(\begin{array}{ccc}0 & i & 0 \\ i & 0 & 0 \\ 0 & 0 & 0\end{array}\right), \quad I_{6}=\frac{1}{2 \sqrt{3}}\left(\begin{array}{ccc}0 & 0 & 0 \\ 0 & 0 & i \\ 0 & i & 0\end{array}\right)$,

$I_{7}=-\frac{i}{2 \sqrt{3}}\left(\begin{array}{ccc}0 & 0 & 0 \\ 0 & 1 & 0 \\ 0 & 0 & -1\end{array}\right), \quad I_{8}=\frac{i}{6}\left(\begin{array}{ccc}2 & 0 & 0 \\ 0 & -1 & 0 \\ 0 & 0 & -1\end{array}\right)$,

corresponding to the antifundamental representation.

The basis elements $\left\{I_{a}, I_{i}\right\}$ of the Lie algebra $\operatorname{su}(3)$ can be represented by left-invariant vector fields $\left\{E_{a}, E_{i}\right\}$ on the Lie group SU(3), and the dual basis $\left\{E^{a}, E^{i}\right\}$ is a set of left-invariant one-forms which obey the Maurer-Cartan equations

$d E^{a}=-f_{j b}^{a} E^{j} \wedge E^{b}-\frac{1}{2} f_{b c}^{a} E^{b} \wedge E^{c}, \quad d E^{i}=-\frac{1}{2} f_{b c}^{i} E^{b} \wedge E^{c}$,

where $i, j=7,8$ correspond to the Cartan subalgebra of $\operatorname{su}(3)$.

Cosets $S U(3) / U(1)_{k, l}$. - Let us consider a U(1) subgroup of $\mathrm{SU}(3)$ given by matrices of the form

$$
h=\left(\begin{array}{ccc}
\exp (i(k+l) \varphi) & 0 & 0 \\
0 & \exp (-i k \varphi) & 0 \\
0 & 0 & \exp (-i l \varphi)
\end{array}\right),
$$

where $k$ and $l$ are relatively prime integers and $0 \leq \varphi \leq 2 \pi$. Consider the coset space

$$
X_{k, l}=\mathrm{SU}(3) / \mathrm{U}(1)_{k, l},
$$

where $\mathrm{U}(1)_{k, l}$ is represented by matrices (2.5). For relatively prime integers $k$ and $l$ the coset spaces $X_{k, l}$ are simply connected manifolds called Aloff-Wallach spaces $[18,19]$.

The space $\mathrm{SU}(3) / \mathrm{U}(1)=: G / H$ consists of left cosets $g H, g \in G$, and the natural projection $g \mapsto g H$ is denoted by

$$
\pi: \mathrm{SU}(3) \rightarrow X_{k, l}
$$

with fibers $\mathrm{U}(1)_{k, l}$. Over a contractible open subset $U$ of $X_{k, l}$, one can choose a map $L: U \rightarrow \mathrm{SU}(3)$ such that $\pi \circ L=\operatorname{Id}_{U}$, i.e. $L$ is a local section of the principal bundle (2.7). The pull-backs of $\left\{E^{a}, E^{i}\right\}$ by $L$ from $\mathrm{SU}(3)$ to $X_{k, l}$ are denoted by $\left\{e^{a}, e^{i}\right\}$ which satisfy the same Maurer-Cartan equations

$$
d e^{a}=-f_{i b}^{a} e^{i} \wedge e^{b}-\frac{1}{2} f_{b c}^{a} e^{b} \wedge e^{c}, \quad d e^{i}=-\frac{1}{2} f_{b c}^{i} e^{b} \wedge e^{c}
$$

as $\left\{E^{a}, E^{i}\right\}$. Note that since all objects we consider will be invariant under some action of SU(3), it will suffice to do calculations just over the subset $U$.

If we denote by $\left\{e^{\hat{a}}\right\}, \hat{a}=1, \ldots, 7$, an orthonormal coframe on $U \subset X_{k, l}$ [basis for $T^{*}\left(X_{k, l}\right)$ over $U$ ] then

$$
\begin{aligned}
e^{\hat{a}} & =e^{a} \quad \text { for } a=1, \ldots, 6, \\
e^{\hat{\jmath}} & =\frac{1}{\Delta}(k+l) e^{7}-\frac{2 \gamma}{\Delta}(k-l) e^{8}
\end{aligned}
$$

with $\left\{e^{a}, e^{i}\right\}$ obeying the Maurer-Cartan equations (2.8) and

$$
e^{\hat{8}}=\frac{1}{2 \gamma \Delta}(k-l) e^{7}+\frac{1}{\Delta}(k+l) e^{8}
$$

is a canonical connection one-form in the bundle (2.7). Here

$$
\gamma:=\frac{1}{2 \sqrt{3}}, \quad \Delta^{2}:=2\left(k^{2}+l^{2}\right) .
$$

Then as generators of $\mathrm{SU}(3)$ we have

$I_{\hat{a}}=I_{a}, \quad I_{\hat{\jmath}}=\Delta^{-1}\left((k+l) I_{7}-\sqrt{3}(k-l) I_{8}\right)$,

$I_{\hat{8}}=\Delta^{-1}\left(\frac{1}{\sqrt{3}}(k-l) I_{7}+(k+l) I_{8}\right)$,

so that

$$
e^{a} I_{a}+e^{i} I_{i}=e^{\hat{a}} I_{\hat{a}}+e^{\hat{8}} I_{\hat{8}},
$$

and $I_{\hat{8}}$ is the generator of the group $\mathrm{U}(1)_{k, l}$ given by (2.5).

Let us now rescale matrices (2.12) as

$$
\begin{gathered}
\tilde{I}_{1}=\gamma^{-1} \varsigma_{1} I_{1}, \quad \tilde{I}_{2}=\gamma^{-1} \varsigma_{1} I_{2}, \quad \tilde{I}_{3}=\gamma^{-1} \varsigma_{2} I_{3}, \\
\tilde{I}_{4}=\gamma^{-1} \varsigma_{2} I_{4}, \quad \tilde{I}_{5}=\gamma^{-1} \varsigma_{3} I_{5}, \quad \tilde{I}_{6}=\gamma^{-1} \varsigma_{3} I_{6}, \\
\tilde{I}_{7}=(\gamma \mu)^{-1} I_{\hat{\gamma}}, \quad \tilde{I}_{8}=(\gamma \mu)^{-1} I_{\hat{8}},
\end{gathered}
$$


such that

$$
e^{a} I_{a}+e^{i} I_{i}=\tilde{e}^{\hat{a}} \tilde{I}_{\hat{a}}+\tilde{e}^{8} \tilde{I}_{8},
$$

and therefore the rescaled coframe fields $\left\{\tilde{e}^{\hat{a}}\right\}$ and the rescaled connection one-form $\tilde{e}^{8}$ have the form

$$
\begin{gathered}
\tilde{e}^{1}=\gamma \varsigma_{1}^{-1} e^{1}, \quad \tilde{e}^{2}=\gamma \varsigma_{1}^{-1} e^{2}, \quad \tilde{e}^{3}=\gamma \varsigma_{2}^{-1} e^{3}, \\
\tilde{e}^{4}=\gamma \varsigma_{2}^{-1} e^{4}, \quad \tilde{e}^{5}=\gamma \varsigma_{3}^{-1} e^{5}, \quad \tilde{e}^{6}=\gamma \varsigma_{3}^{-1} e^{6}, \\
\tilde{e}^{7}=\gamma \mu e^{\hat{\gamma}}, \quad \tilde{e}^{8}=\gamma \mu e^{\hat{8}} .
\end{gathered}
$$

Here we introduced real parameters

$$
\mathrm{s}_{1}, \mathrm{~s}_{2}, \mathrm{~s}_{3}, \mu \in \mathbb{R} \text {. }
$$

As a metric on $X_{k, l}$ we take

$$
d \tilde{s}_{7}^{2}=\delta_{\hat{a} \hat{b}} \tilde{e}^{\hat{a}} \tilde{e}^{\hat{b}} .
$$

One can show that for any given relatively prime integers $k$, $l$ one can choose parameters $\varsigma_{\alpha}$ and $\mu(\alpha=1,2,3)$ such that the metric (2.18) will be Einstein for a connection with a torsion 3-form

$$
\psi=\frac{1}{3 !} \psi_{\hat{a} \hat{b} \hat{c}} \tilde{e}^{\hat{a}} \wedge \tilde{e}^{\hat{b}} \wedge \tilde{e}^{\hat{c}}
$$

having the following nonvanishing components:

$$
\psi_{135}=\psi_{425}=\psi_{416}=\psi_{326}=\psi_{127}=\psi_{347}=\psi_{567}=1 .
$$

Furthermore, this connection has the holonomy group $G_{2}$ and the 3 -form (2.19) defines a $G_{2}$ structure on $X_{k, l}[18,19]$. For more details on the geometry of Aloff-Wallach spaces see e.g. [18-21].

Complex basis on $T^{*}\left(X_{k, l}\right)$.- Note that $X_{1,1}$ can be fibered over the homogeneous manifold $\mathbb{F}_{3}=$ $\mathrm{SU}(3) / \mathrm{U}(1) \times \mathrm{U}(1)$ with fibers

$$
\mathrm{U}(1)^{\perp}=\exp \left(\alpha \tilde{I}_{7}\right)
$$

parametrized by an angle $0 \leq \alpha \leq 2 \pi$. So, for $k=l=1$ we have a projection

$$
X_{1,1} \rightarrow \mathbb{F}_{3}
$$

whose fibers $\mathrm{U}(1)^{\perp}$ are orthogonal complements of $\mathrm{U}(1)=\mathrm{U}(1)_{1,1}$ from (2.5), (2.6), and (2.7) in the torus $T^{2} \cong \mathrm{U}(1) \times \mathrm{U}(1)$ [the Cartan subgroup of $\mathrm{SU}(3)$ ]. This case is very special since $X_{1,1}$ is an Einstein-Sasaki manifold and therefore the cone $\mathcal{C}\left(X_{1,1}\right)$ with the metric

$$
d s_{8}^{2}=d r^{2}+r^{2} d s_{X_{1,1}}^{2}
$$

is a Calabi-Yau 4-conifold with the holonomy group ${ }^{1}$ $\mathrm{SU}(4) \subset \operatorname{spin}(7)$. Furthermore, on $X_{1,1}$ there exists a metric such that $X_{1,1}$ becomes a 3-Sasakian manifold with a hyper-Kähler structure $\operatorname{Sp}(2)$ on the cone $\mathcal{C}\left(X_{1,1}\right)$.

\footnotetext{
${ }^{1}$ Recall that the cone $\mathcal{C}\left(X_{k, l}\right)$ over the general Aloff-Wallach space $X_{k, l}$ has the holonomy group $\operatorname{spin}(7)$.
}

Recall that $\mathbb{F}_{3}$ is fibered over the projective plane $\mathbb{C} P^{2} \cong \mathrm{SU}(3) / \mathrm{U}(2), \mathbb{F}_{3} \rightarrow \mathbb{C} P^{2}$, and the same is true for $X_{k, l}$ with any $k$ and $l$. One can show that fibers of the projection $X_{k, l} \rightarrow \mathbb{C} P^{2}$ are lens spaces $S^{3} / \mathbb{Z}_{p}$ with $p=$ $|k+l|$. For clarity, let us combine all the above fibrations into one diagram

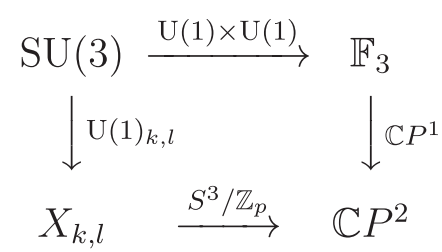

where $X_{k, l}$ can also be fibered over $\mathbb{F}_{3}$ if $k=l=1$.

Note that $S^{3} / \mathbb{Z}_{p}$ is an $S^{1}$-fiber bundle over $\mathbb{C} P^{1}$ and one can consider complex forms which span $\mathbb{C} P^{2}$ and $\mathbb{C} P^{1}$ in $X_{k, l}$ as seen from (2.24). Namely, let us introduce complex one-forms ${ }^{2}$

$\tilde{\Theta}^{1}:=\tilde{e}^{1}+i \tilde{e}^{2}, \quad \tilde{\Theta}^{2}:=\tilde{e}^{3}+i \tilde{e}^{4}, \quad \tilde{\Theta}^{3}=-\tilde{e}^{5}+i \tilde{e}^{6}$

$\tilde{\Theta}^{\overline{1}}:=\tilde{e}^{1}-i \tilde{e}^{2}, \quad \tilde{\Theta}^{\overline{2}}:=\tilde{e}^{3}-i \tilde{e}^{4}, \quad \tilde{\Theta}^{\overline{3}}=-\tilde{e}^{5}-i \tilde{e}^{6}$,

plus real $\tilde{e}^{7}, \tilde{e}^{8}$ and matrices

$\tilde{I}_{1}^{-}:=\frac{1}{2}\left(\tilde{I}_{1}-i \tilde{I}_{2}\right), \quad \tilde{I}_{2}^{-}:=\frac{1}{2}\left(\tilde{I}_{3}-i \tilde{I}_{4}\right), \quad \tilde{I}_{3}^{-}:=\frac{1}{2}\left(-\tilde{I}_{5}-i \tilde{I}_{6}\right)$,

$\tilde{I}_{\overline{1}}^{+}:=\frac{1}{2}\left(\tilde{I}_{1}+i \tilde{I}_{2}\right), \quad \tilde{I}_{\overline{2}}^{+}:=\frac{1}{2}\left(\tilde{I}_{3}+i \tilde{I}_{4}\right), \quad \tilde{I}_{\overline{3}}^{+}:=\frac{1}{2}\left(-\tilde{I}_{5}+i \tilde{I}_{6}\right)$,

$$
-i \tilde{I}_{7},-i \tilde{I}_{8}
$$

which form a basis of the complex Lie algebra $s l(3, \mathbb{C})=$ $s u(3) \otimes \mathbb{C}$. Their explicit form is

$$
\begin{aligned}
& \tilde{I}_{1}^{-}=\varsigma_{1}\left(\begin{array}{lll}
0 & 0 & 0 \\
0 & 0 & 0 \\
1 & 0 & 0
\end{array}\right), \quad \tilde{I}_{\overline{1}}^{+}=\varsigma_{1}\left(\begin{array}{ccc}
0 & 0 & -1 \\
0 & 0 & 0 \\
0 & 0 & 0
\end{array}\right) \text {, } \\
& \tilde{I}_{2}^{-}=\varsigma_{2}\left(\begin{array}{lll}
0 & 1 & 0 \\
0 & 0 & 0 \\
0 & 0 & 0
\end{array}\right), \quad \tilde{I}_{\overline{2}}^{+}=\varsigma_{2}\left(\begin{array}{ccc}
0 & 0 & 0 \\
-1 & 0 & 0 \\
0 & 0 & 0
\end{array}\right) \text {, } \\
& \tilde{I}_{3}^{-}=\varsigma_{3}\left(\begin{array}{lll}
0 & 0 & 0 \\
0 & 0 & 0 \\
0 & 1 & 0
\end{array}\right), \quad \tilde{I}_{\overline{3}}^{+}=\varsigma_{3}\left(\begin{array}{ccc}
0 & 0 & 0 \\
0 & 0 & -1 \\
0 & 0 & 0
\end{array}\right) \text {, } \\
& -i \tilde{I}_{7}=\frac{2}{\mu \Delta}\left(\begin{array}{ccc}
l-k & 0 & 0 \\
0 & -l & 0 \\
0 & 0 & k
\end{array}\right), \quad-i \tilde{I}_{8}=\frac{2}{\mu \Delta \sqrt{3}}\left(\begin{array}{ccc}
k+l & 0 & 0 \\
0 & -k & 0 \\
0 & 0 & -l
\end{array}\right)
\end{aligned}
$$

\footnotetext{
${ }^{2}$ Here, $\tilde{\Theta}^{1,2}$ span the $\mathbb{C} P^{2}$ base in (2.24) and $\tilde{\Theta}^{3}$ spans the $\mathbb{C} P^{1} \hookrightarrow S^{3} / \mathbb{Z}_{p}$, and the choice of the sign in $\tilde{\Theta}^{3}$ is such that an associated almost complex structure on a six-dimensional subbundle of the tangent bundle, defined by $\tilde{\Theta}^{\alpha}, \alpha=1,2,3$, will be integrable. For $\Theta^{3}=\tilde{e}^{5}+i \tilde{e}^{6}$ it will never be integrable. For $k=l=1$ our choice corresponds to a Kähler structure on $\mathbb{F}_{3}$ and $\tilde{\Theta}^{3}=\tilde{e}^{5}+i \tilde{e}^{6}$ corresponds to a nearly Kähler structure on $\mathbb{F}_{3}[9,23]$
} 
We have the commutation relations

$$
\begin{aligned}
{\left[-i \tilde{I}_{j}, \tilde{I}_{\alpha}^{-}\right] } & =\tilde{C}_{j \alpha}^{\beta} \tilde{I}_{\beta}^{-}, \quad\left[-i \tilde{I}_{j}, \tilde{I}_{\bar{\alpha}}^{+}\right]=\tilde{C}_{j \bar{\alpha}}^{\bar{\beta}} \tilde{I}_{\bar{\beta}}^{+}, \\
{\left[\tilde{I}_{\alpha}^{-}, \tilde{I}_{\beta}^{-}\right] } & =\tilde{C}_{\alpha \beta}^{\gamma} \tilde{I}_{\gamma}^{-}, \quad\left[\tilde{I}_{\bar{\alpha}}^{+}, \tilde{I}_{\bar{\beta}}^{+}\right]=\tilde{C}_{\bar{\alpha} \bar{\beta}}^{\bar{\gamma}} \tilde{I}_{\bar{\gamma}}^{+}, \\
{\left[\tilde{I}_{\alpha}^{-}, \tilde{I}_{\bar{\beta}}^{+}\right] } & =\tilde{C}_{\alpha \bar{\beta}}^{j}\left(-i \tilde{I}_{j}\right)+\tilde{C}_{\alpha \bar{\beta}}^{\gamma} \tilde{I}_{\gamma}^{-}+\tilde{C}_{\alpha \bar{\beta}}^{\bar{\gamma}} \tilde{I}_{\bar{\gamma}}^{+},
\end{aligned}
$$

with

$$
\begin{aligned}
& \tilde{C}_{3 \overline{2}}^{1}=-\frac{\varsigma_{2} \varsigma_{3}}{\varsigma_{1}}=-\tilde{C}_{2 \overline{3}}^{\overline{1}}, \quad \tilde{C}_{3 \overline{1}}^{2}=\frac{\varsigma_{3} \varsigma_{1}}{\varsigma_{2}}=-\tilde{C}_{1 \overline{3}}^{\overline{2}}, \\
& \tilde{C}_{12}^{3}=\frac{\varsigma_{1} \varsigma_{2}}{\varsigma_{3}}=\tilde{C}_{\overline{1}}^{\overline{3}}, \quad \tilde{C}_{71}^{1}=\frac{2}{\mu \Delta}(2 k-l)=-\tilde{C}_{7 \overline{1}}^{\overline{1}}, \\
& \tilde{C}_{72}^{2}=\frac{2}{\mu \Delta}(2 l-k)=-\tilde{C}_{7 \overline{2}}^{\overline{2}}, \quad \tilde{C}_{73}^{3}=\frac{2}{\mu \Delta}(k+l)=-\tilde{C}_{7 \overline{3}}^{\overline{3}}, \\
& \tilde{C}_{81}^{1}=-\frac{2}{\sqrt{3} \mu \Delta}(k+2 l)=-\tilde{C}_{8 \overline{1}}^{\overline{1}}, \\
& \tilde{C}_{82}^{2}=\frac{2}{\sqrt{3} \mu \Delta}(2 k+l)=-\tilde{C}_{8 \overline{2}}^{\overline{2}}, \\
& \tilde{C}_{83}^{3}=\frac{2}{\sqrt{3} \mu \Delta}(k-l)=-\tilde{C}_{8 \overline{3}}^{\overline{3}}, \quad \tilde{C}_{1 \overline{1}}^{7}=-\frac{\mu \varsigma_{1}^{2}}{\Delta} k, \\
& \tilde{C}_{2 \overline{2}}^{7}=-\frac{\mu \varsigma_{2}^{2}}{\Delta} l, \quad \tilde{C}_{3 \overline{3}}^{7}=-\frac{\mu \varsigma_{3}^{2}}{\Delta}(k+l), \quad \tilde{C}_{1 \overline{1}}^{8}=\frac{\sqrt{3} \mu \varsigma_{1}^{2}}{\Delta} l, \\
& \tilde{C}_{2 \overline{2}}^{8}=-\frac{\sqrt{3} \mu \varsigma_{2}^{2}}{\Delta} k, \quad \tilde{C}_{3 \overline{3}}^{8}=\frac{\sqrt{3} \mu \varsigma_{3}^{2}}{\Delta}(l-k) .
\end{aligned}
$$

Note that standard undeformed structure constants correspond to $k=l=1, \quad \mu=\gamma^{-1}, \quad \varsigma_{1}=\varsigma_{2}=\gamma$, $\varsigma_{3}=\frac{\gamma}{\sqrt{2}}$, and they are given by

$$
\begin{gathered}
C_{3 \overline{2}}^{1}=-\frac{1}{\sqrt{2}} \gamma=-C_{2 \overline{3}}^{\overline{1}}, \quad C_{3 \overline{1}}^{2}=\frac{1}{\sqrt{2}} \gamma=-C_{1 \overline{3}}^{\overline{2}}, \\
C_{12}^{3}=\sqrt{2} \gamma=C_{\overline{1} \overline{2}}^{\overline{3}}, \quad C_{71}^{1}=C_{72}^{2}=-C_{7 \overline{1}}^{\overline{1}}=-C_{7 \overline{2}}^{\overline{2}}=\gamma, \\
C_{73}^{3}=2 \gamma=-C_{7 \overline{3}}^{\overline{3}}, \quad C_{81}^{1}=-\frac{1}{2}=-C_{8 \overline{1}}^{\overline{1}}, \\
C_{82}^{2}=\frac{1}{2}=-C_{8 \overline{2}}^{\overline{2}}, \quad C_{1 \overline{1}}^{7}=C_{2 \overline{2}}^{7}=C_{3 \overline{3}}^{7}=-\frac{1}{2} \gamma, \\
C_{1 \overline{1}}^{8}=\frac{1}{4}=-C_{2 \overline{2}}^{8} .
\end{gathered}
$$

In the new basis the Maurer-Cartan equations (2.8) become

$$
\begin{aligned}
d \tilde{\Theta}^{\alpha} & =-i \tilde{C}_{j \beta}^{\alpha} \tilde{e}^{j} \wedge \tilde{\Theta}^{\beta}-\frac{1}{2} \tilde{C}_{\beta \gamma}^{\alpha} \tilde{\Theta}^{\beta} \wedge \tilde{\Theta}^{\gamma}-\tilde{C}_{\beta \bar{\gamma}}^{\alpha} \tilde{\Theta}^{\beta} \wedge \tilde{\Theta}^{\bar{\gamma}}, \\
d \tilde{\Theta}^{\bar{\alpha}} & =-i \tilde{C}_{j \bar{\beta}}^{\bar{\alpha}} \tilde{e}^{j} \wedge \tilde{\Theta}^{\bar{\beta}}-\frac{1}{2} \tilde{C}_{\bar{\beta} \bar{\gamma}}^{\bar{\alpha}} \tilde{\Theta}^{\bar{\beta}} \wedge \tilde{\Theta}^{\bar{\gamma}}-\tilde{C}_{\beta \bar{\gamma}}^{\bar{\alpha}} \tilde{\Theta}^{\beta} \wedge \tilde{\Theta}^{\bar{\gamma}}, \\
d \tilde{e}^{j} & =i \tilde{C}_{\beta \bar{\gamma}}^{j} \tilde{\Theta}^{\beta} \wedge \tilde{\Theta}^{\bar{\gamma}},
\end{aligned}
$$

where we have used the structure constants from (2.29). The metric on $X_{k, l}$ in terms of $\tilde{\Theta}^{\alpha}$ and $\tilde{e}^{7}$ is

$$
d \tilde{s}_{7}^{2}=\delta_{\alpha \bar{\beta}} \tilde{\Theta}^{\alpha} \tilde{\Theta}^{\bar{\beta}}+\left(\tilde{e}^{7}\right)^{2},
$$

i.e. we have

$$
g_{\alpha \bar{\beta}}=\frac{1}{2} \delta_{\alpha \bar{\beta}}, \quad g_{77}=1 .
$$

Coset space $X_{1,1}$. - It is known (see e.g. [19]) that for $k=l=1$ the Aloff-Wallach space admits a metric such that the cone $\mathcal{C}\left(X_{1,1}\right)$ over it admits metrics with the holonomy group $\mathrm{SU}(4) \subset \operatorname{spin}(7)$ (Calabi-Yau 4-fold) and $\mathrm{Sp}(2) \subset \mathrm{SU}(4) \subset \operatorname{spin}(7)$ (hyper-Kähler 4-fold). This means that in the Calabi-Yau case on $\mathcal{C}\left(X_{1,1}\right)$ there exists a closed (1,1)-form $\omega^{1,1}$ (Kähler form) and in the hyperKähler case on $\mathcal{C}\left(X_{1,1}\right)$ there exist three Kähler forms:

$$
\omega_{3}=\omega^{1,1}, \quad \omega_{1}, \quad \text { and } \quad \omega_{2},
$$

i.e. besides the closed form $\omega^{1,1}$, we also have a closed $(2,0)$-form $\omega^{2,0}:=\omega_{1}+i \omega_{2}$.

For the general metric $(2.23)$ on $\mathcal{C}\left(X_{1,1}\right)$, one can introduce the $(1,1)$-form as

$$
\omega^{1,1}:=\frac{i}{2} r^{2}\left(\delta_{\alpha \bar{\alpha}} \tilde{\Theta}^{\alpha} \wedge \tilde{\Theta}^{\bar{\alpha}}+\tilde{\Theta}^{4} \wedge \tilde{\Theta}^{\overline{4}}\right),
$$

where

$$
\tilde{\Theta}^{4}:=\frac{d r}{r}-i \tilde{e}^{7}, \quad \tilde{\Theta}^{\overline{4}}:=\frac{d r}{r}+i \tilde{e}^{7} .
$$

We obtain

$$
\begin{aligned}
-2 i d \omega^{1,1}= & \left(2-\mu \varsigma_{1}^{2}\right) \tilde{\Theta}^{1 \overline{1}} \wedge r d r+\left(2-\mu \varsigma_{2}^{2}\right) \tilde{\Theta}^{2 \overline{2}} \wedge r d r \\
& +\left(1-\mu \varsigma_{3}^{2}\right) \tilde{\Theta}^{3 \overline{3}} \wedge 2 r d r \\
& +r^{2}\left(\frac{\varsigma_{2} \varsigma_{3}}{\varsigma_{1}}+\frac{\varsigma_{3} \varsigma_{1}}{\varsigma_{2}}-\frac{\varsigma_{1} \varsigma_{2}}{\varsigma_{3}}\right)\left(\tilde{\Theta}^{12 \overline{3}}-\tilde{\Theta}^{\overline{1} \overline{2} 3}\right),
\end{aligned}
$$

where $\tilde{\Theta}^{\alpha \bar{\beta}}:=\tilde{\Theta}^{\alpha} \wedge \tilde{\Theta}^{\bar{\beta}}$, etc. From (2.37) it follows that $\omega^{1,1}$ is closed if

$$
\varsigma_{1}^{2}=\varsigma_{2}^{2}=2 \varsigma_{3}^{2}=2 \alpha^{2}, \quad \mu=\frac{1}{\alpha^{2}}
$$

for any real number $\alpha$.

$S U(4)$ - and Sp(2)-holonomy on $\mathcal{C}\left(X_{1,1}\right)$. - Note that the closure of the form $\omega^{1,1}$ means that the holonomy group of the cone $\mathcal{C}\left(X_{1,1}\right)$ reduces to the group $\mathrm{U}(4)$ (Kähler structure). For having on $\mathcal{C}\left(X_{1,1}\right)$ a Calabi-Yau structure [SU(4) holonomy] one should impose an additional condition of closure of the $(4,0)$-form

$$
\Omega^{4,0}:=r^{4} \tilde{\Theta}^{1} \wedge \tilde{\Theta}^{2} \wedge \tilde{\Theta}^{3} \wedge \tilde{\Theta}^{4} .
$$

By differentiating (2.39), from the condition $d \Omega^{4,0}=0$ one obtains

$$
\alpha=+1 \quad \text { or } \quad \alpha=-1
$$

that fixes a Calabi-Yau metric on $\mathcal{C}\left(X_{1,1}\right)$. Both $\alpha$ from (2.40) correspond to the same metric on $X_{1,1}$ and the choice of different sign of $\alpha$ corresponds to the choice of different orientation on $X_{1,1}$.

Now we want to check whether this metric allows further reduction of the structure group $\mathrm{SU}(4)$ to the group 
$\mathrm{Sp}(2) \subset \mathrm{SU}(4) \subset \operatorname{spin}(7)$, i.e. allows an introduction of a hyper-Kähler structure on $\mathcal{C}\left(X_{1,1}\right)$. On the Calabi-Yau space $\mathcal{C}\left(X_{1,1}\right)$, we consider the $(2,0)$-form

$$
\omega^{2,0}=r^{2}\left(\tilde{\Theta}^{1} \wedge \tilde{\Theta}^{2}+\beta \tilde{\Theta}^{3} \wedge \tilde{\Theta}^{4}\right),
$$

where $\beta$ is a complex number. Then from the equation $d \omega^{2,0}=0$ we obtain

$$
\beta=\alpha= \pm 1 \text {. }
$$

Therefore, the metric with $\alpha= \pm 1$ from (2.40) allows a hyper-Kähler structure ${ }^{3}$ on the cone $\mathcal{C}\left(X_{1,1}\right)$ and a 3-Sasakian structure on $X_{1,1}$.

\section{SPIN(7) INSTANTONS}

$G_{2}$ instantons and gradient flows. - Consider the ChernSimons-type functional on $X_{k, l}$,

$$
\begin{aligned}
S= & -\frac{1}{4} \int_{X_{k, l}} \operatorname{tr}(\mathcal{F} \wedge \mathcal{F}) \wedge \psi \\
= & -\frac{1}{4} \int_{X_{k, l}} \operatorname{tr}\left(\mathcal{A} \wedge d \mathcal{A}+\frac{2}{3} \mathcal{A} \wedge \mathcal{A} \wedge \mathcal{A}\right) \wedge d \psi \\
& -\frac{1}{4} \int_{X_{k, l}} d\left(\operatorname{tr}\left(\mathcal{A} \wedge d \mathcal{A}+\frac{2}{3} \mathcal{A} \wedge \mathcal{A} \wedge \mathcal{A}\right) \wedge \psi\right),
\end{aligned}
$$

where $\mathcal{A}$ is a connection on a rank-3 complex vector bundle over $X_{k, l}$ [we will specialize to the gauge group $\mathrm{SU}(3)$ in a moment] and $\mathcal{F}=d \mathcal{A}+\mathcal{A} \wedge \mathcal{A}$ is its curvature. For the variation of (3.1) we have

$$
\left(\frac{\delta S}{\delta \mathcal{A}}\right)_{\hat{a}}=\frac{1}{2} *(d \psi \wedge \mathcal{F})_{\hat{a}}=\frac{1}{2} \beta \psi_{\hat{a} \hat{b} \hat{c}} \mathcal{F}_{\hat{b} \hat{c}},
$$

where $*$ is the Hodge operator and $\beta$ is some coefficient which can be calculated. Here, we used the fact that $d \psi \sim * \psi \Rightarrow * d \psi \sim \psi$ on $X_{k, l}$. Therefore, the equations of motion are

$$
d \psi \wedge \mathcal{F}=0 \Leftrightarrow \psi\rfloor \mathcal{F}=0 \Leftrightarrow \psi_{\hat{a} \hat{b} \hat{c}} \mathcal{F}_{\hat{b} \hat{c}}=0 .
$$

Note that (3.3) are exactly $G_{2}$-instanton equations on $X_{k, l}$. Now we can define the Chern-Simons gradient-flow equations

$$
\dot{\mathcal{A}}_{\hat{a}}=\beta^{-1}\left(\frac{\delta S}{\delta \mathcal{A}}\right)_{\hat{a}}=\frac{1}{2} \psi_{\hat{a} \hat{b} \hat{c}} \mathcal{F}_{\hat{b} \hat{c}},
$$

whose stable points $\dot{\mathcal{A}}:=\frac{d \mathcal{A}}{d \tau}=0$ are $G_{2}$ instantons on $X_{k, l}$.

\footnotetext{
${ }^{3}$ Comparing with the standard expression for the symplectic form in Darboux coordinates, the careful reader might notice an unconventional relative sign appearing in (2.41) for the choice $\alpha=-1$. To arrive at the standard expression $\omega^{2,0}=\tilde{\Theta}^{1} \wedge \tilde{\Theta}^{2}+$ $\tilde{\Theta}^{3} \wedge \tilde{\Theta}^{4}$, which is unique up to an overall rescaling, one needs to absorb the sign by replacing $\tilde{\Theta}^{4}$ with minus itself in the definition (2.36). This has no further consequences except for an irrelevant overall sign flip in (2.39) corresponding to the change of orientation.
}

Spin(7)-instanton equations on $\mathbb{R} \times X_{k, l}$. -On the one hand, (3.4) is the flow equation. On the other hand, it is exactly the spin(7)-instanton equation

$$
\mathcal{F}_{0 \hat{a}}=\frac{1}{2} \psi_{\hat{a} \hat{b} \hat{c}} \mathcal{F}_{\hat{b} \hat{c}}
$$

on the space $\mathbb{R} \times X_{k, l}, \tau \in \mathbb{R}$, in the gauge $\mathcal{A}_{\tau} \equiv \mathcal{A}_{0}=0$, where $\tau \equiv x^{0}, d \tau=\tilde{e}^{0}$.

So, let us consider $\mathcal{A}, \mathcal{F} \in s u(3)$, and Eq. (3.5) on the space $\mathbb{R} \times X_{k, l}$. The $\mathrm{SU}(3)$-equivariant Ansatz for $\mathcal{A}$ is

$$
\begin{aligned}
\mathcal{A} & =X_{\hat{a}} \tilde{e}^{\hat{a}}+\tilde{I}_{8} \tilde{e}^{8}=Y_{\alpha} \tilde{\Theta}^{\alpha}+Y_{\bar{\alpha}} \tilde{\Theta}^{\bar{\alpha}}+X_{7} \tilde{e}^{7}+\tilde{I}_{8} \tilde{e}^{8}, \\
\mathcal{A}_{0} & =0,
\end{aligned}
$$

with the following restrictions which guarantee the $\mathrm{SU}(3)$ equivariance:

$$
\left[-i \tilde{I}_{8}, Y_{\alpha}\right]=\tilde{C}_{8 \alpha}^{\beta} Y_{\beta}, \quad\left[-i \tilde{I}_{8}, Y_{\bar{\alpha}}\right]=\tilde{C}_{8 \bar{\alpha}}^{\bar{\beta}} Y_{\bar{\beta}}, \quad\left[\tilde{I}_{8}, X_{7}\right]=0 .
$$

Here

$$
\begin{array}{ll}
Y_{1}:=\frac{1}{2}\left(X_{1}-i X_{2}\right), & Y_{2}:=\frac{1}{2}\left(X_{3}-i X_{4}\right), \\
Y_{3}:=\frac{1}{2}\left(-X_{5}-i X_{6}\right), & Y_{\bar{\alpha}}=-Y_{\alpha}^{\dagger}
\end{array}
$$

are some $3 \times 3$ complex matrices depending on $\tau \in \mathbb{R}, \alpha$, $\beta=1,2,3$.

For (3.6) and (3.7) we have

$$
\begin{aligned}
\mathcal{F}= & \dot{Y}_{\alpha} \tilde{e}^{0} \wedge \tilde{\Theta}^{\alpha}+\dot{Y}_{\bar{\alpha}} \tilde{e}^{0} \wedge \tilde{\Theta}^{\bar{\alpha}}+\dot{X}_{7} \tilde{e}^{0} \wedge \tilde{e}^{7}+\frac{1}{2}\left(\left[Y_{\alpha}, Y_{\beta}\right]\right. \\
& \left.-\tilde{C}_{\alpha \beta}^{\gamma} Y_{\gamma}\right) \tilde{\Theta}^{\alpha} \wedge \tilde{\Theta}^{\beta}+\left(\left[Y_{\alpha}, Y_{\bar{\beta}}\right]-\tilde{C}_{\alpha \bar{\beta}}^{\gamma} Y_{\gamma}-\tilde{C}_{\alpha \bar{\beta}}^{\bar{\gamma}} Y_{\bar{\gamma}}\right. \\
& \left.+i \tilde{C}_{\alpha \bar{\beta}}^{7} X_{7}+i \tilde{C}_{\alpha \bar{\beta}}^{8} \tilde{I}_{8}\right) \tilde{\Theta}^{\alpha} \wedge \tilde{\Theta}^{\bar{\beta}}+\frac{1}{2}\left(\left[Y_{\bar{\alpha}}, Y_{\bar{\beta}}\right]\right. \\
& \left.-\tilde{C}_{\bar{\alpha} \bar{\beta}}^{\bar{\gamma}} Y_{\bar{\gamma}}\right) \tilde{\Theta}^{\bar{\alpha}} \wedge \tilde{\Theta}^{\bar{\beta}}+\left(\left[Y_{\alpha}, X_{7}\right]+i \tilde{C}_{7 \alpha}^{\beta} Y_{\beta}\right) \tilde{\Theta}^{\alpha} \wedge \tilde{e}^{7} \\
& +\left(\left[Y_{\bar{\alpha}}, X_{7}\right]+i \tilde{C}_{7 \bar{\alpha}}^{\bar{\beta}} Y_{\bar{\beta}}\right) \tilde{\Theta}^{\bar{\alpha}} \wedge \tilde{e}^{7},
\end{aligned}
$$

where $\dot{Y}_{\alpha}:=d Y_{\alpha} / d \tau$, etc. We get

$$
\begin{aligned}
& \mathcal{F}_{0 \alpha}=\dot{Y}_{\alpha}, \quad \mathcal{F}_{0 \bar{\alpha}}=\dot{Y}_{\bar{\alpha}}, \quad \mathcal{F}_{07}=\dot{X}_{7}, \\
& \mathcal{F}_{\alpha \beta}=\left[Y_{\alpha}, Y_{\beta}\right]-\tilde{C}_{\alpha \beta}^{\gamma} Y_{\gamma}, \quad \mathcal{F}_{\bar{\alpha} \bar{\beta}}=\left[Y_{\bar{\alpha}}, Y_{\bar{\beta}}\right]-\tilde{C}_{\bar{\alpha} \bar{\beta}}^{\bar{\gamma}} Y_{\bar{\gamma}}, \\
& \mathcal{F}_{\alpha \bar{\beta}}=\left[Y_{\alpha}, Y_{\bar{\beta}}\right]-\tilde{C}_{\alpha \bar{\beta}}^{\gamma} Y_{\gamma}-\tilde{C}_{\alpha \bar{\beta}}^{\bar{\gamma}} Y_{\bar{\gamma}}+i \tilde{C}_{\alpha \bar{\beta}}^{7} X_{7}+i \tilde{C}_{\alpha \bar{\beta}}^{8} \tilde{I}_{8}, \\
& \mathcal{F}_{\alpha 7}=\left[Y_{\alpha}, X_{7}\right]+i \tilde{C}_{7 \alpha}^{\beta} Y_{\beta}, \quad \mathcal{F}_{\bar{\alpha} 7}=\left[Y_{\bar{\alpha}}, X_{7}\right]+i \tilde{C}_{7 \bar{\alpha}}^{\bar{\beta}} Y_{\bar{\beta}} .
\end{aligned}
$$

Reduction to matrix equations. - Note that

$$
\begin{aligned}
\psi & =\frac{1}{3 !} \psi_{\hat{a} \hat{b} \hat{c}} \tilde{e}^{\hat{a} \hat{b} \hat{c}} \\
& =\psi_{12 \overline{3}} \tilde{\Theta}^{12 \overline{3}}+\psi_{\overline{1} \overline{2} 3} \tilde{\Theta}^{\overline{1} \overline{2} 3}+\frac{1}{2 !} \psi_{7 \alpha \bar{\beta}} \tilde{e}^{7} \wedge \tilde{\Theta}^{\alpha \bar{\beta}},
\end{aligned}
$$

and therefore 


$$
\psi_{12 \overline{3}}=\psi_{\overline{1} \overline{2} 3}=-\frac{1}{2}, \quad \psi_{71 \overline{1}}=\psi_{72 \overline{2}}=\frac{i}{2}, \quad \psi_{73 \overline{3}}=-\frac{i}{2} .
$$

Thus, from (3.5) we have

$$
\begin{gathered}
2 \mathcal{F}_{0 \alpha}=-2 \psi_{\alpha \beta \bar{\gamma}} \mathcal{F}_{\gamma \bar{\beta}}+\psi_{\alpha \bar{\beta} \bar{\gamma}} \mathcal{F}_{\beta \gamma}+2 \psi_{\alpha \bar{\beta} \bar{\gamma}} \mathcal{F}_{\beta 7}, \\
2 \mathcal{F}_{0 \bar{\alpha}}=2 \psi_{\bar{\alpha} \bar{\beta} \gamma} \mathcal{F}_{\beta \bar{\gamma}}+\psi_{\bar{\alpha} \beta \gamma} \mathcal{F}_{\bar{\beta} \bar{\gamma}}+2 \psi_{\bar{\alpha} \beta \bar{\gamma}} \mathcal{F}_{\bar{\beta} 7}, \\
2 \mathcal{F}_{07}=\psi_{7 \hat{a} \hat{b}} \mathcal{F}_{\hat{a} \hat{b}}=-2 \psi_{7 \alpha \bar{\alpha}} \mathcal{F}_{\alpha \bar{\alpha}} .
\end{gathered}
$$

Substituting (3.10), (3.11), and (3.12) into (3.13), (3.14), and (3.15), we obtain the following matrix equations:

$$
\begin{aligned}
2 \dot{Y}_{\overline{1}} & =\left(\tilde{C}_{2 \overline{3}}^{\overline{1}}+\tilde{C}_{7 \overline{1}}^{\overline{1}}\right) Y_{\overline{1}}+\left[i X_{7}, Y_{\overline{1}}\right]-\left[Y_{2}, Y_{\overline{3}}\right], \\
2 \dot{Y}_{\overline{2}} & =\left(\tilde{C}_{\overline{3} 1}^{\overline{2}}+\tilde{C}_{7 \overline{2}}^{\overline{2}}\right) Y_{\overline{2}}+\left[i X_{7}, Y_{\overline{2}}\right]-\left[Y_{\overline{3}}, Y_{1}\right], \\
2 \dot{Y}_{\overline{3}} & =\left(\tilde{C}_{\overline{1} \overline{2}}^{\overline{3}}-\tilde{C}_{7 \overline{3}}^{\overline{3}}\right) Y_{\overline{3}}-\left[i X_{7}, Y_{\overline{3}}\right]-\left[Y_{\overline{1}}, Y_{\overline{2}}\right], \\
2 \dot{X}_{7} & =\tilde{C}^{8} \tilde{I}_{8}+\tilde{C}^{7} X_{7}-i\left(\left[Y_{1}, Y_{\overline{1}}\right]+\left[Y_{2}, Y_{\overline{2}}\right]-\left[Y_{3}, Y_{\overline{3}}\right]\right),
\end{aligned}
$$

where

$$
\tilde{C}^{8}:=\tilde{C}_{1 \overline{1}}^{8}+\tilde{C}_{2 \overline{2}}^{8}-\tilde{C}_{3 \overline{3}}^{8}, \quad \tilde{C}^{7}:=\tilde{C}_{1 \overline{1}}^{7}+\tilde{C}_{2 \overline{2}}^{7}-\tilde{C}_{3 \overline{3}}^{7} .
$$

All structure constants in (3.16), (3.17), (3.18), (3.19), and (3.20) can be taken from (2.29). The above matrix equations can be written concisely by means of a "superpotential" $W$ via

$$
\dot{Y}_{\bar{\alpha}}=\delta_{\bar{\alpha} \beta} \frac{\partial W}{\partial Y_{\beta}}, \quad \dot{X}_{7}=\frac{\partial W}{\partial X_{7}} .
$$

The explicit form of the superpotential $W\left(Y_{1}, Y_{2}, Y_{3}, Y_{\overline{1}}, Y_{\overline{2}}, Y_{\overline{3}}, X_{7}\right)$,

$$
\begin{aligned}
W= & \frac{1}{2} \operatorname{tr}\left\{\left(\tilde{C}_{2 \overline{3}}^{\overline{1}}+\tilde{C}_{7 \overline{1}}^{\overline{1}}\right) Y_{1} Y_{\overline{1}}+\left(\tilde{C}_{\overline{3} 1}^{\overline{2}}+\tilde{C}_{\overline{7}}^{\overline{2}}\right) Y_{2} Y_{\overline{2}}\right. \\
& +\left(\tilde{C}_{\overline{1} \overline{3}}^{\overline{3}}-\tilde{C}_{7 \overline{3}}\right) Y_{3} Y_{\overline{3}}-\left[Y_{\overline{2}}, Y_{3}\right] Y_{\overline{1}}-\left[Y_{\overline{3}}, Y_{1}\right] Y_{2} \\
& +i\left(\left[X_{7}, Y_{\overline{1}}\right] Y_{1}+\left[X_{7}, Y_{\overline{2}}\right] Y_{2}-\left[X_{7}, Y_{\overline{3}}\right] Y_{3}\right) \\
& \left.+\tilde{C}^{8} \tilde{I}_{8} X_{7}+\frac{1}{2} \tilde{C}^{7}\left(X_{7}\right)^{2}\right\},
\end{aligned}
$$

follows by inspection of (3.16), (3.17), (3.18), (3.19), and (3.20). It can also be obtained directly by inserting the Ansatz (3.6) into the Chern-Simons-type action (3.1).

Reduction to equations on scalar fields of $\tau$.-The SU (3)-equivariance conditions (3.7) are solved by

$Y_{\overline{1}}=\bar{\phi}^{\overline{1}} \tilde{I}_{\overline{1}}, \quad Y_{\overline{2}}=\bar{\phi}^{2} \tilde{I}_{\overline{2}}, \quad Y_{\overline{3}}=\bar{\phi}^{\overline{3}} \tilde{I}_{\overline{3}}, \quad Y_{1}=\phi^{1} \tilde{I}_{1}$,

$Y_{2}=\phi^{2} \tilde{I}_{2}, \quad Y_{3}=\phi^{3} \tilde{I}_{3}, \quad X_{7}=\chi^{7} \tilde{I}_{7}+\chi^{8} \tilde{I}_{8}$,

where $\phi^{\alpha}(\alpha=1,2,3)$ are complex scalar fields depending on $\tau$, and $\chi^{i}(i=7,8)$ are real scalar fields of $\tau$.
Substituting (3.23) into (3.16), (3.17), (3.18), and (3.19), we obtain

$2 \dot{\phi}^{1}=\left(\tilde{C}_{2 \overline{3}}^{\overline{1}}+\tilde{C}_{7 \overline{1}}^{\overline{1}}-\chi^{7} \tilde{C}_{7 \overline{1}}^{\overline{1}}-\chi^{8} \tilde{C}_{8 \overline{1}}^{\overline{1}}\right) \phi^{1}-\tilde{C}_{2 \overline{3}}^{\overline{1}} \bar{\phi}^{\overline{2}} \phi^{3}$,

$2 \dot{\phi}^{2}=\left(\tilde{C}_{\overline{3} 1}^{\overline{2}}+\tilde{C}_{7 \overline{2}}^{\overline{2}}-\chi^{7} \tilde{C}_{7 \overline{2}}^{\overline{2}}-\chi^{8} \tilde{C}_{8 \overline{2}}^{\overline{2}}\right) \phi^{2}-\tilde{C}_{\overline{3} 1}^{\overline{2}} \bar{\phi}^{\overline{1}} \phi^{3}$,

$2 \dot{\phi}^{3}=\left(\tilde{C}_{\overline{1} \overline{2}}^{\overline{3}}-\tilde{C}_{7 \overline{3}}^{\overline{3}}+\chi^{7} \tilde{C}_{7 \overline{3}}^{\overline{3}}+\chi^{8} \tilde{C}_{8 \overline{3}}^{\overline{3}}\right) \phi^{3}-\tilde{C}_{\overline{1} \overline{2}}^{\overline{3}} \phi^{1} \phi^{2}$,

$2 \dot{\chi}^{7}=\tilde{C}^{7} \chi^{7}-\tilde{C}_{1 \overline{1}}^{7}\left|\phi^{1}\right|^{2}-\tilde{C}_{2 \overline{2}}^{7}\left|\phi^{2}\right|^{2}+\tilde{C}_{3 \overline{3}}^{7}\left|\phi^{3}\right|^{2}$,

$2 \dot{\chi}^{8}=\tilde{C}^{8}+\tilde{C}^{7} \chi^{8}-\tilde{C}_{1 \overline{1}}^{8}\left|\phi^{1}\right|^{2}-\tilde{C}_{2 \overline{2}}^{8}\left|\phi^{2}\right|^{2}+\tilde{C}_{3 \overline{3}}^{8}\left|\phi^{3}\right|^{2}$,

where $\tilde{C}^{7}$ and $\tilde{C}^{8}$ are given in (3.20). The superpotential $W$ becomes

$$
\begin{aligned}
2 W= & -\varsigma_{1}^{2}\left(\tilde{C}_{2 \overline{3}}^{\overline{1}}+\tilde{C}_{7 \overline{1}}^{\overline{1}}\right)\left|\phi^{1}\right|^{2}-\varsigma_{2}^{2}\left(\tilde{C}_{\overline{3} 1}^{\overline{2}}+\tilde{C}_{7 \overline{2}}^{\overline{2}}\right)\left|\phi^{2}\right|^{2} \\
& -\varsigma_{3}^{2}\left(\tilde{C}_{\overline{1} \overline{2}}^{\overline{3}}-\tilde{C}_{7 \overline{3}}^{\overline{3}}\right)\left|\phi^{3}\right|^{2}+\varsigma_{1} \varsigma_{2} \varsigma_{3}\left(\phi^{1} \phi^{2} \bar{\phi}^{\overline{3}}+\bar{\phi}^{\overline{1}} \bar{\phi}^{\overline{2}} \phi^{3}\right) \\
& +\left(\varsigma_{1}^{2} \tilde{C}_{7 \overline{1}}^{\overline{1}}\left|\phi^{1}\right|^{2}+\varsigma_{2}^{2} \tilde{C}_{7 \overline{2}}^{\overline{2}}\left|\phi^{2}\right|^{2}-\varsigma_{3}^{2} \tilde{C}_{7 \overline{3}}^{\overline{3}}\left|\phi^{3}\right|^{2}\right) \chi^{7} \\
& +\left(\varsigma_{1}^{2} \tilde{C}_{8 \overline{1}}^{\overline{1}}\left|\phi^{1}\right|^{2}+\varsigma_{2}^{2} \tilde{C}_{8 \overline{2}}^{\overline{2}}\left|\phi^{2}\right|^{2}-\varsigma_{3}^{2} \tilde{C}_{8 \overline{3}}^{\overline{3}}\left|\phi^{3}\right|^{2}\right) \chi^{8} \\
& -\tilde{C}^{8} K_{8 i} \chi^{i}-\frac{1}{2} \tilde{C}^{7} K_{i j} \chi^{i} \chi^{j},
\end{aligned}
$$

where $K$ is the Killing metric $K(I, J)=-\operatorname{tr}(I, J)$ for the rescaled generators (2.27). The necessity to introduce $K$ is due to the fact that $\tilde{I}_{7}$ and $\tilde{I}_{8}$ are not mutually orthogonal for general values of $k$ and $l$. The explicit form of $K$ is given by

$K_{\alpha \bar{\beta}}=-\operatorname{tr}\left(\tilde{I}_{\alpha} \tilde{I}_{\bar{\beta}}\right)=\varsigma_{\alpha}^{2} \delta_{\alpha \bar{\beta}} \quad($ no sum over $\alpha)$,

$$
\begin{aligned}
& K_{77}=-\operatorname{tr}\left(\tilde{I}_{7} \tilde{I}_{7}\right)=\frac{8\left(k^{2}-k l+l^{2}\right)}{\mu^{2} \Delta^{2}}, \\
& K_{88}=-\operatorname{tr}\left(\tilde{I}_{8} \tilde{I}_{8}\right)=\frac{8\left(k^{2}+k l+l^{2}\right)}{3 \mu^{2} \Delta^{2}}, \\
& K_{78}=-\operatorname{tr}\left(\tilde{I}_{7} \tilde{I}_{8}\right)=-\frac{4(k-l)(k+l)}{\sqrt{3} \mu^{2} \Delta^{2}}
\end{aligned}
$$

with all other components vanishing. We are now in a position to express the first-order equations (3.24) in terms of the superpotential $W$,

$$
\dot{\phi}^{\alpha}=-K^{\alpha \bar{\beta}} \frac{\partial W}{\partial \bar{\phi}^{\bar{\beta}}}, \quad \dot{\chi}^{i}=-K^{i j} \frac{\partial W}{\partial \chi^{j}} .
$$

The nonvanishing components of the inverse Killing metric are given by

$$
\begin{aligned}
K^{\alpha \bar{\beta}} & =\varsigma_{\alpha}^{-2} \delta^{\alpha \bar{\beta}}, & K^{77} & =-\frac{\mu^{4}}{4} K_{88}, \\
K^{88} & =-\frac{\mu^{4}}{4} K_{77}, & K^{78} & =\frac{\mu^{4}}{4} K_{78},
\end{aligned}
$$

such that $K^{\alpha \bar{\beta}} K_{\gamma \bar{\beta}}=\delta_{\gamma}^{\alpha}, K^{\alpha \bar{\beta}} K_{\alpha \bar{\gamma}}=\delta_{\bar{\gamma}}^{\bar{\beta}}$, and $K^{i j} K_{j k}=\delta_{k}^{i}$.

Equation (3.24) is a complicated set of coupled, nonlinear first-order ordinary differential equations and finding the general solution is a formidable task. Instead, one may 
consider simplifications of these equations by setting some of the fields to zero and hoping to find explicit solutions for these special cases. Indeed, Eq. (3.24) admits a particularly simple yet important special solution, namely,

$$
\begin{gathered}
\phi^{1}=\phi^{2}=\phi^{3}=\chi^{7}=0, \\
\chi^{8}(\tau)= \begin{cases}-\frac{\tilde{C}^{8}}{\tilde{C}}+A \cdot \exp \left(\frac{\tilde{C}^{7}}{2} \tau\right), & \text { if } \tilde{C}^{7} \neq 0, \\
\frac{\tilde{C}^{8}}{2} \tau+B, & \text { if } \tilde{C}^{7}=0,\end{cases}
\end{gathered}
$$

where $A, B \in \mathbb{R}$ are constants of integration. For $\tilde{C}^{7} \neq 0$, $A=0$, this solution is stationary and corresponds to the Abelian (rescaled, if $\tilde{C}^{8} \neq 0$ ) canonical connection on a line bundle over $X_{k, l}$. This is arguably the simplest example for a $G_{2}$ instanton on Aloff-Wallach spaces. A similar conclusion also holds for $\tilde{C}^{7}=\tilde{C}^{8}=0$ with the rescaled canonical connection corresponding to the case $B \neq 0$.

Before specializing to $k=l=1$, we briefly mention that the second-order equations of motion and the potential $V$ for the scalar fields can be obtained straightforwardly from the above first-order equations by simply applying another time derivative to (3.27). The result can be written as

$$
\ddot{\phi}^{\alpha}=K^{\alpha \bar{\beta}} \frac{\partial V}{\partial \bar{\phi}^{\bar{\beta}}}, \quad \ddot{\chi}^{i}=K^{i j} \frac{\partial V}{\partial \chi^{j}} .
$$

The potential $V$ is determined by the usual formula in terms of the superpotential

$$
V=K^{\alpha \bar{\beta}} W_{\alpha} W_{\bar{\beta}}+\frac{1}{2} K^{i j} W_{i} W_{j},
$$

where we introduced the shorthand notation $W_{\alpha}=$ $\partial W / \partial \phi^{\alpha}, \quad W_{\bar{\beta}}=\partial W / \partial \bar{\phi}^{\bar{\beta}}, \quad$ and $\quad W_{i}=\partial W / \partial \chi^{i}$. Computing the gradient of $V$ yields

$$
\begin{aligned}
V_{\alpha} & =K^{\beta \bar{\gamma}}\left(W_{\alpha \beta} W_{\bar{\gamma}}+W_{\alpha \bar{\gamma}} W_{\beta}\right)+K^{i j} W_{\alpha i} W_{j}, \\
V_{i} & =K^{\alpha \bar{\beta}}\left(W_{i \alpha} W_{\bar{\beta}}+W_{i \bar{\beta}} W_{\alpha}\right)+K^{j k} W_{i j} W_{k} .
\end{aligned}
$$

From this and (3.31) we can read off that critical points of the superpotential are both zeros and critical points of the potential. On the other hand, the critical points of $V$ fall into two categories: zero-energy ones $(V=0)$ and positive-energy ones $(V>0)$. The former are precisely the critical points of $W$, which will be studied further for the special case $k=l=1$ in the remainder of this section. However, the positive-energy critical points of $V$ do not correspond to critical points of $W$. Instead, for them the gradient of $W$ is a "zero eigenvector" of the Hessian of $W$. They will not play a role in our analysis.

Specialization to $k=l=1$.- For the special case of $X_{1,1}$, with $\varsigma_{\alpha}$ and $\mu$ given in (2.38), from (3.24) we obtain
$2 \dot{\phi}^{1}=\left(\alpha-1+\chi^{7}-\sqrt{3} \chi^{8}\right) \phi^{1}-\alpha \bar{\phi}^{\overline{2}} \phi^{3}$,

$2 \dot{\phi}^{2}=\left(\alpha-1+\chi^{7}+\sqrt{3} \chi^{8}\right) \phi^{2}-\alpha \bar{\phi}^{\overline{1}} \phi^{3}$,

$\dot{\phi}^{3}=\left(\alpha+1-\chi^{7}\right) \phi^{3}-\alpha \phi^{1} \phi^{2}$,

$2 \dot{\chi}^{7}=-\chi^{7}+\left|\phi^{1}\right|^{2}+\left|\phi^{2}\right|^{2}-\left|\phi^{3}\right|^{2}$,

$2 \dot{\chi}^{8}=-\chi^{8}-\sqrt{3}\left|\phi^{1}\right|^{2}+\sqrt{3}\left|\phi^{2}\right|^{2}$,

with $\alpha= \pm 1$ for the 3-Sasakian structure on $X_{1,1}$. The Killing metric in this case becomes diagonal with nonzero components

$$
K_{1 \overline{1}}=K_{2 \overline{2}}=2 K_{3 \overline{3}}=K_{77}=K_{88}=2 .
$$

The superpotential simplifies to

$$
\begin{aligned}
W= & (1-\alpha)\left(\left|\phi^{1}\right|^{2}+\left|\phi^{2}\right|^{2}\right)-(1+\alpha)\left|\phi^{3}\right|^{2} \\
& +\frac{1}{2}\left(\left(\chi^{7}\right)^{2}+\left(\chi^{8}\right)^{2}\right)+\alpha\left(\phi^{1} \phi^{2} \bar{\phi}^{\overline{3}}+\bar{\phi}^{\overline{1}} \bar{\phi}^{\overline{2}} \phi^{3}\right) \\
& -\left(\left|\phi^{1}\right|^{2}+\left|\phi^{2}\right|^{2}-\left|\phi^{3}\right|^{2}\right) \chi^{7}+\sqrt{3}\left(\left|\phi^{1}\right|^{2}-\left|\phi^{2}\right|^{2}\right) \chi^{8}
\end{aligned}
$$

and (3.33) may be written as

$$
\begin{gathered}
2 \dot{\phi}^{1}=-W_{\overline{1}}, \quad 2 \dot{\phi}^{2}=-W_{\overline{2}}, \quad 2 \dot{\phi}^{3}=-2 W_{\overline{3}}, \\
2 \dot{\chi}^{7}=-W_{7}, \quad 2 \dot{\chi}^{8}=-W_{8} .
\end{gathered}
$$

The superpotential (3.35) is invariant under global $\mathrm{U}(1) \times$ $\mathrm{U}(1)$ transformations of the form

$$
\begin{aligned}
& \left(\phi^{1}, \phi^{2}, \phi^{3}\right) \mapsto\left(e^{i \delta_{1}} \phi^{1}, e^{i \delta_{2}} \phi^{2}, e^{i \delta_{3}} \phi^{3}\right) \quad \text { with } \\
& \delta_{1}+\delta_{2}-\delta_{3}=0 \bmod 2 \pi .
\end{aligned}
$$

Note that the phases of the $\phi^{\alpha}$ only enter in the cubic terms $\left(\phi^{1} \phi^{2} \bar{\phi}^{\overline{3}}+\bar{\phi}^{\overline{1}} \bar{\phi}^{\overline{2}} \phi^{3}\right)$ in the superpotential, which are thus proportional to $\cos \left(\arg \phi^{1}+\arg \phi^{2}-\arg \phi^{3}\right)$. The superpotential is extremized when $\arg \phi^{1}+\arg \phi^{2}-\arg \phi^{3}=0$ or $\pi$ and, together with (3.37), this allows us to consider purely real fields when searching for extrema of $W$. After fixing $\phi^{\alpha} \in \mathbb{R}$, there is a residual symmetry which acts by flipping the sign of any two of the three complex functions $\phi^{\alpha}$. Therefore, we can restrict ourselves not only to real fields but also take, for example, $\phi^{1}$ and $\phi^{2}$ non-negative when searching for extrema of $W$.

In addition, there is a $Z_{2}$ symmetry which acts by interchanging $\phi^{1}$ and $\phi^{2}$ accompanied by a sign flip of $\chi^{8}$

$$
\left(\phi^{1}, \phi^{2}, \chi^{8}\right) \mapsto\left(\phi^{2}, \phi^{1},-\chi^{8}\right) .
$$

Explicit solutions for $k=l=1$.-We begin by finding the extrema of the superpotential (3.35). Making use of the argument given at the end of the previous section, we take all fields to be real and $\phi^{1}, \phi^{2}$ non-negative. We then need to solve the following equations in five real variables: 


$$
\begin{aligned}
\left(\alpha-1+\chi^{7}-\sqrt{3} \chi^{8}\right) \phi^{1}-( \pm) \alpha \phi^{2} \phi^{3} & =0 \\
\left(\alpha-1+\chi^{7}+\sqrt{3} \chi^{8}\right) \phi^{2}-( \pm) \alpha \phi^{1} \phi^{3} & =0 \\
\left(\alpha+1-\chi^{7}\right) \phi^{3}-( \pm) \alpha \phi^{1} \phi^{2} & =0 \\
-\chi^{7}+\left(\phi^{1}\right)^{2}+\left(\phi^{2}\right)^{2}-\left(\phi^{3}\right)^{2} & =0 \\
-\chi^{8}-\sqrt{3}\left(\phi^{1}\right)^{2}+\sqrt{3}\left(\phi^{2}\right)^{2} & =0
\end{aligned}
$$

The sign ambiguity in the first three equations is a consequence of $\cos \left(\arg \phi^{1}+\arg \phi^{2}-\arg \phi^{3}\right)= \pm 1$ at the extrema. The last two equations may be used to immediately eliminate $\chi^{7}$ and $\chi^{8}$ and one is then left with three cubic equations for three unknowns,

$$
\begin{aligned}
\left(\alpha-1+4\left(\phi^{1}\right)^{2}-2\left(\phi^{2}\right)^{2}-\left(\phi^{3}\right)^{2}\right) \phi^{1}-( \pm) \alpha \phi^{2} \phi^{3} & =0, \\
\left(\alpha-1+4\left(\phi^{2}\right)^{2}-2\left(\phi^{1}\right)^{2}-\left(\phi^{3}\right)^{2}\right) \phi^{2}-( \pm) \alpha \phi^{1} \phi^{3} & =0, \\
\left(\alpha+1-\left(\phi^{1}\right)^{2}-\left(\phi^{2}\right)^{2}+\left(\phi^{3}\right)^{2}\right) \phi^{3}-( \pm) \alpha \phi^{1} \phi^{2} & =0 .
\end{aligned}
$$

One obvious solution is $\phi^{\alpha}=\chi^{i}=0$. However, the full analysis depends on the choice of $\alpha=+1$ or -1 . The results for $\alpha=+1$ are summarized in the following table:

\begin{tabular}{ccccccc}
$\phi^{1}$ & $\phi^{2}$ & $\phi^{3}$ & $\phi^{7}$ & $\phi^{8}$ & Eigenvalues of Hessian & $W$ \\
\hline 0 & 0 & 0 & 0 & 0 & $(-,+,+, 0,0)$ & 0 \\
1 & 1 & \pm 1 & 1 & 0 & $(-,-,+,+,+)$ & $-1 / 2$
\end{tabular}

where the sign ambiguity in the $\phi^{3}$ column stems from the fact that $\cos \left(\arg \phi^{1}+\arg \phi^{2}-\arg \phi^{3}\right)= \pm 1$ at the extrema [cf. Eq. (3.39)]. There is one saddle point and a degenerate critical point at the origin. The appearance of the degenerate critical point can be understood from a physics perspective by noticing that $\phi^{1}$ and $\phi^{2}$ are massless and therefore correspond to flat directions in the space of solutions.

The results for $\alpha=-1$ are summarized in the following table:

\begin{tabular}{ccccccc}
$\phi^{1}$ & $\phi^{2}$ & $\phi^{3}$ & $\phi^{7}$ & $\phi^{8}$ & $\begin{array}{c}\text { Eigenvalues } \\
\text { of Hessian }\end{array}$ & $W$ \\
\hline 0 & 0 & 0 & 0 & 0 & $(+,+,+,+, 0)$ & 0 \\
$1 / \sqrt{2}$ & 0 & 0 & $1 / 2$ & $-\sqrt{3} / 2$ & $(-,+,+,+,+)$ & $1 / 2$ \\
$c_{+}$ & $c_{-}$ & $\pm 2 / 3$ & $1 / 6$ & $-\sqrt{35} / 6$ & $(-,-,+,+,+)$ & $31 / 54$ \\
$2 \sqrt{2} / 3$ & $2 \sqrt{2} / 3$ & $\pm 2 / 3$ & $4 / 3$ & 0 & $(-,-,+,+,+)$ & $40 / 27$ \\
1 & 1 & \pm 1 & 1 & 0 & $(-,-,-,+,+)$ & $3 / 2$
\end{tabular}

where $c_{ \pm}=\frac{1}{6} \sqrt{11 \pm \sqrt{105}}$, and again the sign ambiguity in the $\bar{\phi}^{3}$ column is due to the fact that $\cos \left(\arg \phi^{1}+\right.$ $\left.\arg \phi^{2}-\arg \phi^{3}\right)= \pm 1$ at the extrema. The origin is a degenerate critical point. The field $\phi^{3}$ is massless and hence corresponds to a flat direction in the space of solutions, which explains why the critical point at the origin is degenerate. In addition, there are four isolated saddle points.

A few remarks are in order concerning the critical points found above. First of all, we note that the critical point at the origin (i.e. where all scalar fields vanish) corresponds to the Abelian canonical connection on a rank-3 complex vector bundle over $X_{1,1}$ and is thus arguably the simplest explicit example of a $G_{2}$ instanton on an Aloff-Wallach space. Also, the point where $\chi^{8}=0$ and all other scalar fields are equal to unity corresponds to a flat connection $\mathcal{F}=0$. These observations are valid for both choices of $\alpha=+1$ or -1 .

Now that we have found the critical points of the superpotential, we consider the gradient flow connecting suitable pairs of them. In other words, we look for solutions of (3.33), which start at $\tau=-\infty$ from a critical point with a larger value of $W$ and flow as $\tau \rightarrow \infty$ toward a critical point with a smaller value of $W$. These kink configurations are finite-action solutions of (3.5) and thus allow a physical interpretation as $\operatorname{spin}(7)$ instantons on $\mathbb{R} \times X_{1,1}$.

In the search for instanton solutions, one is immediately faced with two technical difficulties. First, the structure of the equations which needs to be solved is such that conventional analytic methods (and known exact solution Ansätze) are not applicable. For example, the well-known hyperbolic tangent-type kink solutions, which inter alia work in one dimension lower [13], do not respect the structure of (3.33). This means we need to resort to numerical methods.

Second, with the exception of the degenerate critical point at the origin, all other critical points are isolated saddle points. Solutions flowing toward these points are unstable. For a given starting point, there is exactly one trajectory whose end point is an isolated saddle point and it is crucial to pick the initial direction to be exactly along this unique trajectory. Combined with the first point, this presents us with a numerical "fine-tuning problem" when it comes to choosing the correct initial conditions for the desired flow. One would somehow need to know the trajectory's direction at the starting point before even attempting to (numerically) solve the equations, leaving oneself with a "fishing in the dark" situation. Moreover, even the smallest deviation from the correct direction will lead to solutions which, instead of approaching the saddle point, will roll off to $\pm \infty$ swiftly.

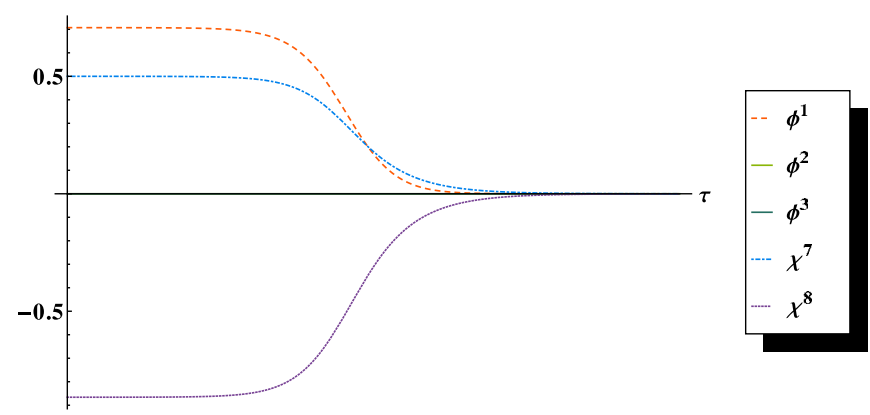

FIG. 1 (color online). Kink solution for $\alpha=-1$ flowing from $W=1 / 2$ at $\tau=-\infty$ to $W=0$ as $\tau \rightarrow \infty . \phi^{2}$ and $\phi^{3}$ are zero everywhere and thus their plot coincides with the $\tau$ axis. 
There is only one case where this fine-tuning problem does not occur and where we have been able to find an explicit (numerical) solution. It is the kink solution for $\alpha=$ -1 flowing from $W=1 / 2$ at $\tau=-\infty$ to $W=0$ as $\tau \rightarrow \infty$. The numerical solution for this case is shown in Fig. 1. It should be noted that the shape of these curves resembles that of a hyperbolic tangent-type kink. Indeed, although a hyperbolic tangent Ansatz does not solve Eqs. (3.33), it does provide a good approximation. The maximal deviation from the actual numerical solution is of the order of $1 \%$.

\section{ACKNOWLEDGMENTS}

We thank Derek Harland for helpful comments. This work was supported in part by the cluster of excellence EXC 201 "Quantum Engineering and Space-Time Research," by the Deutsche Forschungsgemeinschaft (DFG), by the Russian Foundation for Basic Research (Grant No. RFBR 09-02-91347), and by the HeisenbergLandau program.
[1] M. B. Green, J.H. Schwarz, and E. Witten, Superstring Theory (Cambridge University Press, Cambridge, UK, 1987).

[2] E. Corrigan, C. Devchand, D. B. Fairlie, and J. Nuyts, Nucl. Phys. B214, 452 (1983).

[3] R. S. Ward, Nucl. Phys. B236, 381 (1984).

[4] S. K. Donaldson, Proc. London Math. Soc. s3-50, 1 (1985); S. K. Donaldson, Duke Math. J. 54, 231 (1987); K. K. Uhlenbeck and S.-T. Yau, Commun. Pure Appl. Math. 39, S257 (1986).

[5] M. Mamone Capria and S. M. Salamon, Nonlinearity 1, 517 (1988); R. Reyes Carrión, Diff. Geom. Appl. 8, 1 (1998).

[6] L. Baulieu, H. Kanno, and I. M. Singer, Commun. Math. Phys. 194, 149 (1998).

[7] G. Tian, Ann. Math. 151, 193 (2000); T. Tao and G. Tian, J. Am. Math. Soc. 17, 557 (2004).

[8] S. K. Donaldson and R.P. Thomas, The Geometric Universe (Oxford University Press, Oxford, New York, 1998); S. K. Donaldson and E. Segal, arXiv:0902.3239.

[9] A. D. Popov, Lett. Math. Phys. 92, 253 (2010); D. Harland and A. D. Popov, arXiv:1005.2837; A. D. Popov and R. J. Szabo, arXiv:1009.3208.

[10] D. B. Fairlie and J. Nuyts, J. Phys. A 17, 2867 (1984); S. Fubini and H. Nicolai, Phys. Lett. 155B, 369 (1985); T. A. Ivanova and A. D. Popov, Lett. Math. Phys. 24, 85 (1992); Theor. Math. Phys. 94, 225 (1993); M. Günaydin and H. Nicolai, Phys. Lett. B 351, 169 (1995).

[11] O. Lechtenfeld, A. D. Popov, and R. J. Szabo, J. High Energy Phys. 12 (2003) 022; A. D. Popov, A.G. Sergeev, and M. Wolf, J. Math. Phys. (N.Y.) 44, 4527 (2003); O. Lechtenfeld, A. D. Popov, and R. J. Szabo, J. High Energy Phys. 08 (2008) 093.
[12] T. A. Ivanova, O. Lechtenfeld, A. D. Popov, and T. Rahn, Lett. Math. Phys. 89, 231 (2009); T. Rahn, J. Math. Phys. (N.Y.) 51, 072302 (2010).

[13] D. Harland, T. A. Ivanova, O. Lechtenfeld, and A. D. Popov, Commun. Math. Phys. 300, 185 (2010); I. Bauer, T. A. Ivanova, O. Lechtenfeld, and F. Lubbe, J. High Energy Phys. 10 (2010) 044.

[14] H. N. Sà Earp, Ph.D. thesis, Imperial College London, 2009.

[15] C. Lewis, Ph.D. thesis, Oxford University, 1998.

[16] M. Grana, Phys. Rep. 423, 91 (2006); M. R. Douglas and S. Kachru, Rev. Mod. Phys. 79, 733 (2007); R. Blumenhagen, B. Kors, D. Lüst, and S. Stieberger, Phys. Rep. 445, 1 (2007).

[17] A. Strominger, Nucl. Phys. B274, 253 (1986); C. M. Hull, Phys. Lett. 167B, 51 (1986); Phys. Lett. B 178, 357 (1986); D. Lüst, Nucl. Phys. B276, 220 (1986); B. de Wit, D. J. Smit, and N. D. Hari Dass, Nucl. Phys. B283, 165 (1987).

[18] S. Aloff and N. Wallach, Bull. Am. Math. Soc. 81, 93 (1975).

[19] F. M. Cabrera, M. D. Monar, and A. F. Swann, J. Lond. Math. Soc. 53, 407 (1996); Th. Friedrich, I. Kath, A. Moroianu, and U. Semmelmann, J. Geom. Phys. 23, 259 (1997); Hong Van Le and M. Munir, arXiv:0912.0169.

[20] M. Cvetic, G. W. Gibbons, H. Lu, and C. N. Pope, Nucl. Phys. B617, 151 (2001); Phys. Rev. D 65, 106004 (2002); Y. Konishi and M. Naka, Classical Quantum Gravity 18, 5521 (2001).

[21] H. Kanno and Y. Yasui, J. Geom. Phys. 43, 293 (2002); 43, 310 (2002); A. Bilal, J.P. Derendinger, and K. Sfetsos, Nucl. Phys. B628, 112 (2002).

[22] S. Gukov and J. Sparks, Nucl. Phys. B625, 3 (2002); G. Curio, B. Kors, and D. Lüst, Nucl. Phys. B636, 197 (2002).

[23] A. D. Popov, Nucl. Phys. B828, 594 (2010). 